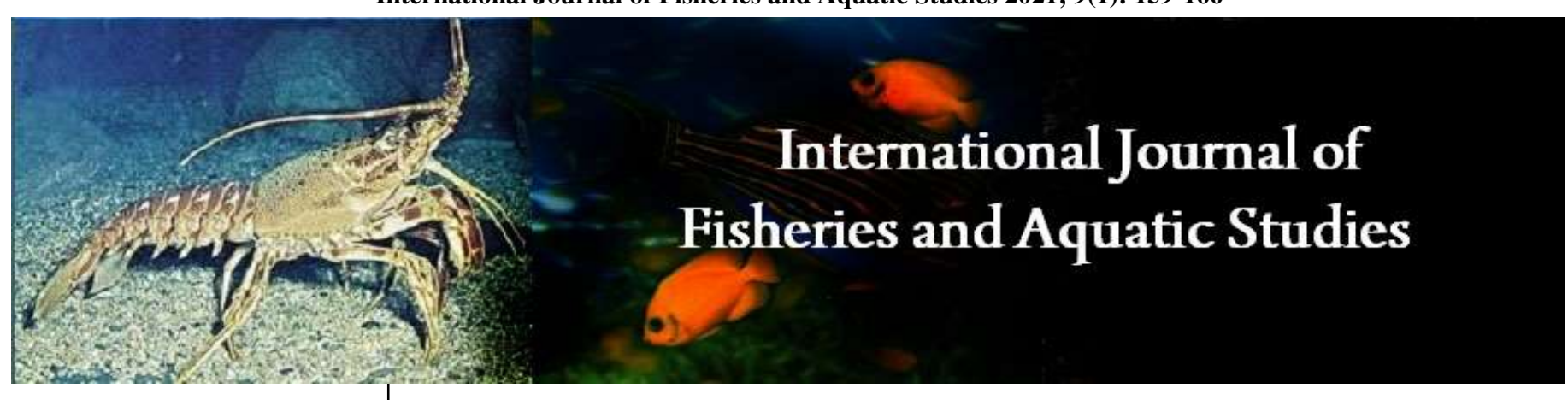

E-ISSN: 2347-5129

P-ISSN: 2394-0506

(ICV-Poland) Impact Value: 5.62

(GIF) Impact Factor: 0.549

IJFAS 2021; 9(1): 159-166

(C) 2021 IJFAS

www.fisheriesjournal.com

Received: 19-11-2020

Accepted: 30-12-2020

Ravitchandirane Vaithilingam

Kanchi Mamunivar Government

Institute for Postgraduate

Studies and Research,

Puducherry, India

Kavipriya Jeacoumar

Kanchi Mamunivar Government

Institute for Postgraduate

Studies and Research,

Puducherry, India

Nithya Mary Srinivasan

Kanchi Mamunivar Government

Institute for Postgraduate

Studies and Research,

Puducherry, India

Vijilakshmi Udhyasuriyan Kanchi Mamunivar Government

Institute for Postgraduate

Studies and Research,

Puducherry, India
Corresponding Author:

Ravitchandirane Vaithilingam

Kanchi Mamunivar Government

Institute for Postgraduate

Studies and Research,

Puducherry, India

\section{Checklist of commercially important fishes of Puducherry coastal waters, east coast of India}

\author{
Ravitchandirane Vaithilingam, Kavipriya Jeacoumar, Nithya Mary \\ Srinivasan and Vijilakshmi Udhyasuriyan
}

DOI: $\underline{\text { https://doi.org/10.22271/fish.2021.v9.i1b.2400 }}$

\begin{abstract}
The aim of this study is to update the list of fish species in order to increase the knowledge about biodiversity of commercially important fishes of Puducherry coastal waters. This study also concentrated on the availability and threatened status of commercially important fish species. A total of 88 fish species under 36 families and 11 orders has been collected during the study. Order Perciformes were made up to 65\% with 23 families and 58 species followed by Clupeidae 15\% with 3 families and 13 species. Clupeiformes stand on top in the catch followed by perches and mackerel's. The study revealed that there were noteworthy variations found in the ichthyofaunal diversity. Among the 36 families, only 27 families were seen in the caught without any depletion. Order Beloniformes and Gadiformes showed sudden appearance in the catch which were not seen for the past 7 years. IUCN status of 88 species were studied, about $63 \%$ of species were under least concern and $8 \%$ near threatened, $3 \%$ vulnerable category, $7 \%$ data deficient, $19 \%$ not evaluated. Anthropogenic activities affect aquatic habitats leading to loss of many species as well as bring changes in the species composition. Therefore, ichthyofaunal diversity studies on regular basis are essential for sustainable management.
\end{abstract}

Keywords: Ichthyofaunal diversity, fishery production, iucn status, conservation, Puducherry

\section{Introduction}

Every realm of the sea is a warehouse of biodiversity which is a residence for multifarious of life. Having a deep understanding of biodiversity is an essential element for survival for most of the human past. The description of new species and mapping of their distribution is an important activity in post-enlightenment science (Costello et al. 2013) ${ }^{[1]}$. Among various living organisms, fishes are most diverse vertebrate occupying various habitats in different types of ecosystem. In terms of biodiversity fishes overtake all other group of organisms (Mustafa S.1999) ${ }^{[2]}$. Fish are immensely important to human beings as they have long been a staple food item for a longer period. The Indian fisheries sector plays a valuable role in the global fish production and it is the second largest producer of fish in the world. The importance of the fisheries sector in India is revealed by the fact that it employs more than five million people (Anon, 2000) ${ }^{[3]}$, contributes to food and nutritional security and employment, supports livelihoods and raises the socioeconomic status of poor fishing communities. Fishes are involved in the environmental policies as biodiversity and ecological quality indicators (Kestemont et.al.,2000,Schmutz et al.,2007) ${ }^{[4,5]}$ and they have been used successfully in biogeographical studies, ecoregion delineations (Abell et al.,2008) [6], conservation evaluations(Moyle and Randall.,1998) ${ }^{[7]}$ and assessments of ecologically acceptable water regime management(Jowett.,1997) ${ }^{[8]}$. The marine fish diversity of India is in ever-increasing danger due to overfishing of commercially important species. Further, fish may be considered as apt indicators of aquatic biodiversity, since their rich diversity is reflective of a wide range of environmental conditions (Moyle PB, Leidy RA.1992) ${ }^{[9]}$. It is well known that biodiversity is in world-wide decline (Butchart et al. 2010) ${ }^{[10]}$. Knowledge of fish diversity of the particular region is considered to be essential not only for their rational management but also for the conservational strategies for the ichthyo-fauna of that region. Considering the above, the present study has been made to provide a well-documented checklist about species composition and IUCN status of commercially important ichthyofaunal diversity of Puducherry coastal waters. 


\section{Materials and method}

\subsection{Study area}

Puducherry has a coastline of $45 \mathrm{~km}$, with a continental shelf area of about $1000 \mathrm{sq} . \mathrm{km}$ and it lies between North Latitudes $11^{\circ} 46^{\prime}$ and 120 03' and East Latitudes $79^{\circ} 52^{\prime}$ bounded by Bay of Bengal on the East. The diversity study was conducted in the 14 fish landing centres of Puducherry viz. Kanagachettikulam, Periyakalapet, Chinnakalapet, Pillaichavadi, Solainagar, Vaithikuppam, Kuruchikuppam, Thengaithittu, Veerampattinam, Pudukuppam, Nallavadu, Narambai, Pannithitu and Moorthikuppam from 2016 to 2017.

\subsection{Fish collection methods}

The collections was done almost on daily basis during morning and evening hours. The major objective of the bio inventory is to identify all the available species in the habitat using all gear combinations. Information regarding the seasonal availability, fish catch, size, quality, value of fish, common name, crafts and gears used etc., has been collected from traditional fishermen of all the coastal villages and recorded. Collected fish samples were brought to the laboratory and preserved in $10 \%$ formaldehyde and identification of the species was established by using FAO identification sheets (Fischer and Bianchi, 1984) ${ }^{[11]}$.

\subsection{Gear combinations}

Various types of fishing gears are used by the fishermen of Puducherry region for exploiting different types of fishes. The major part of marine fish landings in Puducherry was from mechanised sector $(86.7 \%)$. Motorised and non-motorised sectors contributed $13.3 \%$ and $0.04 \%$ respectively (Grinson George et al. 2016) ${ }^{[12]}$. The motorized sector includes gillnets, seine nets, hooks \& lines and bagnets. All small boats and catamarans with low sea worthiness take on single day fishing with unfixed voyage times based on the season and the fishery resource which they target. Multiday and single day trawlers together contributed $82 \%$ of the total catch (FRAD, CMFRI, 2017) ${ }^{[13]}$.

\section{Results}

A total of 88 commercially important fish species belonging to 36 families and 11 orders were reported from all landing centres of Puducherry. Out of 88 species, 84 species were teleost and the remaining were cartilaginous (Table: 1). In general, the fishes belong to the class Actinopterygii were found to be dominant. The best represented order was Perciformes $(65 \%)$ with 23 families and 58 species followed by Clupeidae (15\%) with 3 families and 13 species. Other families were each represented by 1 to 3 species (Fig: 1).Among 88 species, 40 species were caught regularly (45\%), 33 species were less available (38\%), 9 species shows rare occurrence $(10 \%)$ and 6 species (Hilsha ilisha, Hilsha other, Harpodon nehereus, Eel, Bregmaceros, Elagatis) were very rare (7\%) (Fig.2). In present study, as per IUCN 2016 red list status, out of 88 species, about $63 \%$ of species were under the status of least concern and $8 \%$ near threatened, 3\% vulnerable category, $7 \%$ data deficient, $19 \%$ not evaluated categories (Fig. 3).

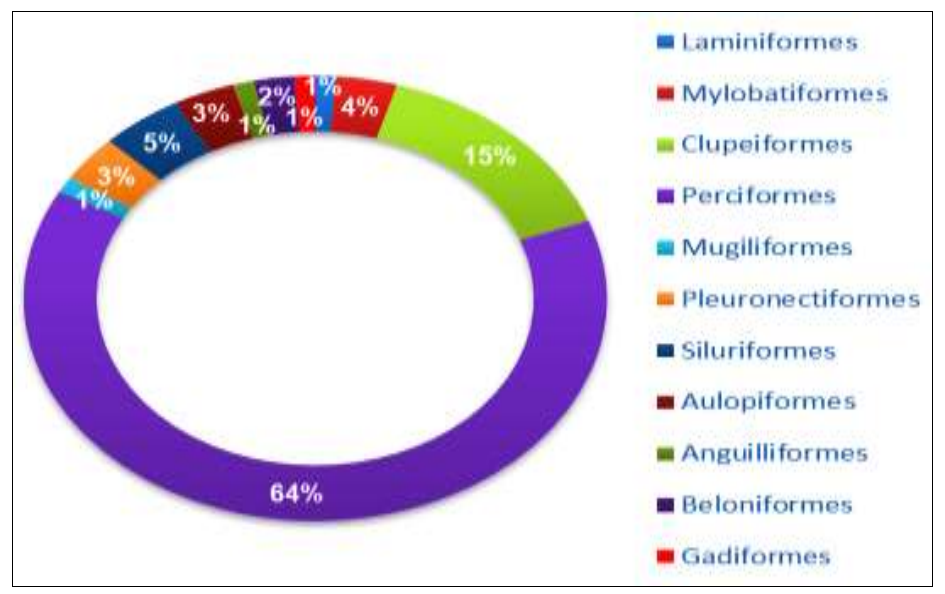

Fig 1: Order wise representation of fish in the study area

\section{Discussion}

The focus of the present study is to establish the list of commercially important fish species with its IUCN status. In this study visual illustrations and descriptive statistics were used to study the fish diversity in Puducherry. Among fin fishes, the pelagic group was dominant which contributed about $70.3 \%$ of the total catch followed by demersal fin fish (29.6\%). The major fish species representing the fish landings along Puducherry coast were sardines including oil sardines, Indian mackerel, seer fish, pomfrets, carangids, perches (sea bass and Leiognathus), flat fishes, mullets, crockers, etc. Many researchers have tried to foretell the more availability of pelagic resources, particularly oil sardine, from the relationship between catches and climatic as well as oceanographic features such as seawater temperature, salinity, rainfall, upwelling and chlorophyll concentration (Banse, 1959; Longhurstand Wooster, 1990; Madhupratap et al., 1994; Yohannan and Abdulrahiman, 1998; Jayaprakash, 2002; Xu and Boyce, 2009) [14, 15, 16, 17, 18, 19]. Order
Perciformes and Clupeiformes held most of the families and species (Table: 1). A total of 23 families falls under Perciformes in which the Carangidae family was dominated by holding 11 species followed by Scombridae with 9 species. Family Sciaenidae and Polynemidae were also contributed with a reasonable of 5 species each. 13 species were recorded in order Clupeiformes under three families. Out of this, Clupeidae family was represented by 7 species. These observation were common in most of marine environment. The presence of large number of species in these two orders could also be explained by the fact that they found favourable condition which allows them to develop and grow (Leveque et al., 1991) ${ }^{[20]}$. The present finding was supported by Kumaran et al., (2012) ${ }^{[21]}$ in Griyampeta estuary, Yanam and Kuppan et al., (2016) ${ }^{[22]}$ from east coastal region, Chennai. Among the 36 families, only 27 families were seen in the caught without any depletion (Puducherry Fisheries Statistics, 2010) ${ }^{[23]}$. It is made known that, six fish groups (Hilsha ilisha, Hilsha other, Harpodon nehereus, Eel, Bregmaceros, 
Elagatis) were in downfall status owing to human activities such as over fishing, modification of water quality etc.(Konan Gervias et al., 2014) ${ }^{[24]}$. It has been came to know that 2 groups viz, Belone \& Hemiramphus and Lactarius were resurface again in the catch during 2015 to 2017 which were not seen before 2015 (Puducherry Fisheries Statistics, 2017) [25].

Among the total 88 species reported during the study, 40 species were available (45\%), 33 species were less available $(38 \%), 9$ species were rare $(10 \%)$ and 6 species were very rare (7\%) (Fig.2) based on the availability status recorded by Department of Fisheries and Fishermen Welfare, Puducherry. From the various detailed studies on fishery resources reported by the scientists of CMFRI (2006) ${ }^{[26]}$, it is understood that $65 \%$ of the commercially important fish varieties in marine waters are overfished. Over exploitation indicates that fisheries prompted changes in the ecosystem owing to low productivity of the coastal waters and high density of fishing craft. The number of mechanised and motorised vessels went amassed due to ultimate demand for seafood and subsequent price escalation. Various developmental programmes of Central and State Governments such as subsidies for diesel engines, new gears and gear materials, vessels and financial assistance to fishermen and cooperative societies (Srivastava et al., 1991) ${ }^{[27]}$ zipped up the modernization in fishing process. Present estimated number of fishing vessels of almost all the types seem to be far excess than the actual number required to produce the sustainable yield (Mohammed Kasim and Vivekanandan, CMFRI, 2011) ${ }^{[28]}$.In simple words, coastal biodiversity is at risk and marine environments are threatened (Imtiyaz et al., 2011) ${ }^{[29]}$ due to intensified human activities (Dulvy et al. 2014) ${ }^{[30]}$.

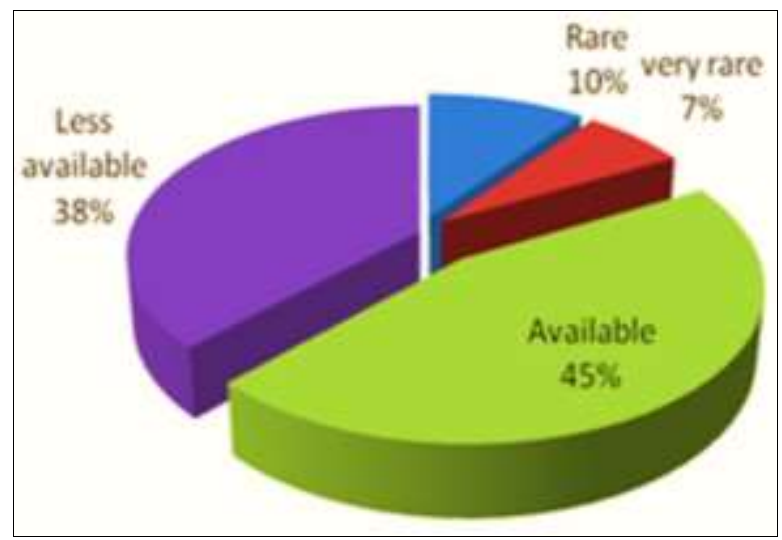

Fig 2: Availability of recorded fish species in the study area

\subsection{Threatened Status}

While evaluating the threat status of 88 fish species, 6 species comes under near threatened (NT), 3 species are vulnerable, 53 least concern, 5 species have deficient data and data about 17 species did not drop under any threat category so it was placed in not evaluated group (Table 1). In present study, as per IUCN $2016^{(31)}$ red list status out of 88 species, about $63 \%$ of species were under least concern and $8 \%$ near threatened, $3 \%$ vulnerable category, $7 \%$ data deficient, $19 \%$ not evaluated categories (Fig. 3).

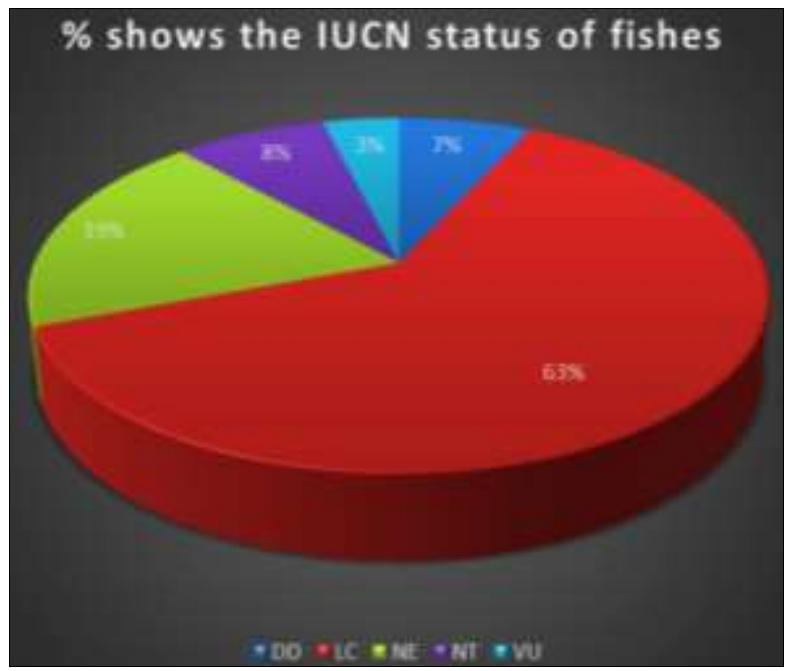

Fig: 3: IUCN stat us of fishes

The coastal waters of Puducherry receive discharges from 4 major industrial complexes including untreated municipal sewage from urban settlements and tourist resorts (Ramachandran, 2001) ${ }^{[32]}$. The other source of marine pollution includes tourism activities which lead to dumping of plastic and other solid wastes by tourists on the beaches.
Recreational activities like tourism lead to loss of habitats, habitat degradation, the spread of disease, pollution, and unsustainable fishing practices are directly related to the actions of humans and recovery from these problems is rarely straightforward. 
Table: 1: Systematic position of the fishes collected in the study with threat status

Super class: pisces

Class:1: chondrichthyes

Subclass:1:elasmobranchii

Super order: Gnathostomata Order and suborder

Order 1: Lamniformes

suborder: Scyliorhinoidei

Order 2: Myliobatiformes Suborder: Squloidae

Class 2: Osteichthyes

Subclass 2: actinopterygii

Super order: Teloestei

\begin{tabular}{|c|c|c|}
\hline Family \& subfamily & & Name of species \\
\hline Family 1: Carcharhinidae & 1 & Rhizoprionodon acutus, Rüppell, 1837 \\
\hline & & \\
\hline Family 2: Dasyatidae & 2 & Dasyatis zugei, Müller \& Henle, 1841 \\
\hline Subfamily: Dasyatinae & 3 & Himantura uarnak, Gmelin, 1789 \\
\hline & 4 & Dasyatis jenkinsii, Annandale, 1909 \\
\hline
\end{tabular}

\begin{tabular}{|c|c|}
\hline Common Name & Vern \\
\hline Milk shark & \\
\hline Pale-edged stingray & \\
\hline Honey combed stingray & Pul \\
\hline Sharpnose stingray & Se
\end{tabular}

\begin{tabular}{|c|c|}
\hline Vernacular name & IUCN/status \\
\hline Pall surTa & LC/AV \\
\hline
\end{tabular}

Chambara-kah Pulli- thirukkai

Sharpnose stingray

Sen-thirukkai

\begin{tabular}{|c|c|}
\hline Family 3: Clupeidae & 5 \\
\hline Subfamily: Clupeinae & 6 \\
\hline Subfamily: Alosinae & 7 \\
\hline & 8 \\
\hline Subfamily: Dorosomatinae & 10 \\
\hline Subfamily: Pristigasterinae & 11 \\
\hline Family 4: Engraulidae & \\
\hline Subfamily: Collinae & 12 \\
\hline Subfamily: Engraulinae & 13 \\
\hline & 14 \\
\hline & 15 \\
\hline Family 5: Chirocentridae & 16 \\
\hline & 17 \\
\hline Family 6: Centropomidae & 18 \\
\hline Family 7: Serranidae & \\
\hline Subfamily: Epinephelinae & 19 \\
\hline Family 8: Terapontidae & 20 \\
\hline & 21 \\
\hline Family 9: Lutjanidae & 22 \\
\hline Family 10: Gerreidae & 23 \\
\hline Family 11: Lethrinidae & 24 \\
\hline Family 12: Nemipteridae & 25 \\
\hline Family:13 Priacanthidae & 26 \\
\hline Family:14 Rachycentridae & 27 \\
\hline Family 15: Siganidae & 28 \\
\hline Family 16: Sillaginidae & 29 \\
\hline Family 17: Lactariidae & 30 \\
\hline Family 18: Carangidae & 32 \\
\hline
\end{tabular}

$5 \quad$ Sardinella longiceps Valenciennes, 1847

Thryssa vitrirostris, Gilchrist \& Thompson, 1908
19 Epinephelus diacanthus, Valenciennes, 1828 Terapon jarbua Forsskal, 1775 Terapon theraps, Cuvier, 1829

21 Lutjanus argentimaculatus, Forsskal, 1775
Gerres filamentosus, Cuvier, 1829 Lethrinus nebulosus, Forsskål, 1775 Nemipterus japonicus, Bloch, 1791 Priacanthus hamrur,Forsskal, 1775 Rachicentron canadum, Linnaeus, 1766 Siganus javus, Linnaeus, 1766 Sillago sihama, Forsskal, 1775

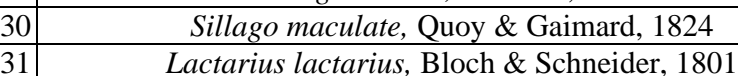
$32 \quad$ Caranx para, Cuvier, 1833

\begin{tabular}{|c|c|c|}
\hline Indian oil sardine & Mathi & LC/AV \\
\hline White sardine & Therakuthuva & LC/AV \\
\hline White Sardinella & Thattakavalai & LC/AV \\
\hline Hilsa shad & Karuvaullam & LC/VR \\
\hline Toli shad & Chudai & LC/VR \\
\hline Chacunda gizzard & Poikendai & LC/AV \\
\hline Shad Tardoore & Thalporuva & LC/AV \\
\hline Golden anchovy & Thova & LC/LA \\
\hline Commerson's anchovy & Therangunni & LC/LA \\
\hline Moustached thryssa & Poruva & LC/LA \\
\hline Orange mouth thryssa & Nedunporuva & LC/LA \\
\hline White fin wolf herring & Mullvalai & LC/R \\
\hline Dorab wolf herring & Karivalai & $\mathrm{LC} / \mathrm{R}$ \\
\hline Sea bass & Koduva & NE/AV \\
\hline Thorny cheek groupers & Kadisan & NT/AV \\
\hline Jarbua Terapon & Katta keetchan & LC/AV \\
\hline Large scaled terapon & Thova keetchan & LC/AV \\
\hline Mangrove red snapper & Ertha & LC/AV \\
\hline Whip fin silver biddy & Oodan & LC/AV \\
\hline Spangled emperor & Kal & LC/AV \\
\hline Japanese threadfin bream & Sankara & NE/AV \\
\hline Moontail bulls eye & Seruppu & LC/AV \\
\hline Cobia & Kadaveerang & LC/AV \\
\hline Streaked spine foot & Oora & LC/AV \\
\hline Silver sillago & Vella kezhangan & LC/AV \\
\hline Trumpeter sillago & Karupu kezhangan & LC/AV \\
\hline False travelly & Sudumbu & NE/R \\
\hline Banded scad & Ottampaara & LC/AV \\
\hline
\end{tabular}

Order 3: Clupeiformes

Suborder: Clupeoidae

Escualosa thoracata, Valenciennes, 1847

Sardinella albella, Valenciennes, 1847

Hilsha ilisha, Hamilton\& Buch., 1822

Hilsa toli, Valenciennes, 1847

Anodontostoma chacunda, Hamilton, 1822

Ophisthopterus tardoore, Cuvier, 1829

Colia dussumieri, Valenciennes, 1848

Stolephorus commersonii, Lacepède, 1803 Thryssa mystax, Schneider, 1801

Chirocentrus nudus, Swainson, 1839

Order 4: Perciformes

Suborder: Percoidei

Chirocentrus dorab, Forsskål, 1775

\author{
18 \\ Lates calcarifer, Bloch, 1790
}

\begin{tabular}{ll}
\hline \\
\hline
\end{tabular}

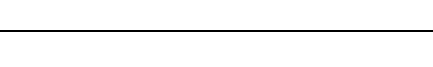
$+2 \cos$ (2)

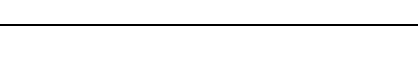
(20)

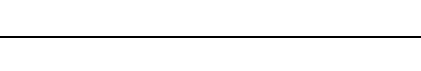
Family 18: Carangidae 


\begin{tabular}{|c|c|c|c|c|c|c|}
\hline & & 33 & Chorinemus lysan, Forsskål, 1775 & Double spotted queen fish & & $\mathrm{DD} / \mathrm{R}$ \\
\hline & & 34 & Elagatis bipinnulata, Quoy \& Gaimard, 1825 & Rainbow runner & Kulkul & LC/VR \\
\hline & & 35 & Alepes djedaba, Forsskål, 1775 & Shrimp scad & Valam paarai & LC/LA \\
\hline & & 36 & Atropus atropos Bloch \& Schneider, 1801 & leftbally travelly & Thallam paarai & NE/LA \\
\hline & & 37 & Atule mate, Cuvier, 1833 & Yellow tail scad & Manja paarai & LC/LA \\
\hline & & 38 & Carangoides chrysophrys, Valenciennes, 1833 & Large nose travelly & Vezhum paarai & LC/LA \\
\hline & & 39 & Gnathanodon speciosus, Forsskål, 1775 & Golden travelly & Eera paarai & $\mathrm{LC} / \mathrm{LA}$ \\
\hline & & 40 & Decapterus russelli, Ruppell, 1830 & Indian scad & Kilitchai & LC/LA \\
\hline & & 41 & Megalaspis cordyla, Linnaeus, 1758 & Torpedo scad & Valangadai & LC/LA \\
\hline & & 42 & Trachinotus mookalee, Cuvier, 1832 & Indian pompano & Perum paarai & LC/LA \\
\hline & & 44 & Leiognathus brevirostris, Valenciennes, 1835 & Short nose pony fish & Oola kaarai & NE/AV \\
\hline & & 45 & Gazza minuta, Bloch, 1795 & Toothed pony fish & Sudupana kaarai & LC/AV \\
\hline & Family 20: Scianidae & 46 & Dendrophysa russelli, Cuvier, 1830 & Goat croaker & Paruva thaalai & LC/LA \\
\hline & & 47 & Johnius belangerii, Cuvier, 1830 & Belangers croaker & Thur kathalai & NE/LA \\
\hline & & 48 & Nibea maculata, Schneider, 1801 & Blotched croaker & $\begin{array}{c}\text { Kaarimunch } \\
\text { kathalai }\end{array}$ & $\mathrm{NE} / \mathrm{LA}$ \\
\hline & & 49 & Protonibea diacanthus, Lacepède, 1802 & Spotted croaker & Panna & NE/LA \\
\hline & & 50 & Otolithoides biauritus, Cantor, 1849 & Bronze croaker & Kaasai kathalai & NE/LA \\
\hline & Family 21: Mullidae & 51 & Upeneus moluccensis, Bleeker, 1855 & Gold banned goatfish & Kuliri & LC/AV \\
\hline \multirow[t]{2}{*}{ Suborder: Sphyraenoidei } & Family 23: Sphyraenidae & 53 & Sphyraena jello, Cuvier, 1829 & Pick handle barracuda & Seela & NE/LA \\
\hline & & 54 & Sphyraena obtusata, Cuvier, 1829 & Obtuse barracuda & Arakola & NE/LA \\
\hline \multirow[t]{5}{*}{ Suborder: Polynemoidei } & Family 24: Polynemidae & 55 & Eleutheronema tetradactylum, Shaw, 1804 & Four finger thread fin & Kittikaala & $\mathrm{DD} / \mathrm{R}$ \\
\hline & & 56 & Polynemus heptadactylus, Cuvier, 1829 & Seven finger thread fin & Erakaala & NE/R \\
\hline & & 57 & Polynemus indicus, Shaw, 1804 & Indian thread fin & Vaalangkaala & $\mathrm{LC} / \mathrm{R}$ \\
\hline & & 58 & Polynemus paradiseus, Linnaeus, 1758 & Paradise thread fin & Thadikaala & $\mathrm{LC} / \mathrm{R}$ \\
\hline & & 59 & Polynemus sextarius, Bloch \& Schneider, 1801 & Black spot thread fin & Pazhaikaala & $\mathrm{NE} / \mathrm{R}$ \\
\hline \multirow[t]{12}{*}{ Suborder: Scombridei } & Family 25: Trichiuridae & 60 & Trichiurus lepturus, Linnaeus, 1758 & Large head hair tail & Chunnambu vaalai & $\mathrm{LC} / \mathrm{LA}$ \\
\hline & Family 26: Scombridae & 61 & Scomberomorus commerson, Lacepède, 1802) & Spanish mackerel & \begin{tabular}{|c|} 
Maavalasi \\
\end{tabular} & NT/AV \\
\hline & & 62 & Euthynnus affinis, Cantor, 1850 & Kawa kawa & Kakka churai & LC/AV \\
\hline & & 63 & Rastrelliger kanagurta, Cuvier, 1816 & Indian mackerel & Kanangeluthi & DD/AV \\
\hline & & 64 & Scomberomorus guttatus, Bloch \& Schneider, 1801 & Indopacific king mackerel & Pulli vanjeeram & DD/AV \\
\hline & & 65 & Auxis thazard, Lacepède, 1803 & Frigate tuna & Veldra churai & LC/AV \\
\hline & & 66 & Katsuwonus pelamis, Linnaeus, 1758 & Skipjack tuna & Vari churai & LC/AV \\
\hline & & 67 & Thunnus albacares, Bonnaterre, 1788 & Yellow fin tuna & Manja churai & NT/AV \\
\hline & & 68 & Thunnus obesus, Lowe, 1839 & Big eye tuna & Sundam & VU/AV \\
\hline & & 69 & Thunnus tonggol, Bleeker, 1851 & Long tail tuna & Sundam & $\mathrm{DD} / \mathrm{AV}$ \\
\hline & Family 27: Stromateidae & 70 & Pampus argenteus, Euphrasen, 1788 & Silver pomfrets & Vella vauva & $\mathrm{LC} / \mathrm{LA}$ \\
\hline & & 71 & Pampus chinensis, Euphrasen, 1788 & Chinese silver pomfret & Tharattai & NE/LA \\
\hline \multicolumn{7}{|l|}{ Order Order:5 Mugiliformes } \\
\hline Suborder: Mugiloidei & Family 22: Mugilidae & 74 & Mugil cephalus, Linnaeus, 1758 & Flat head mullet & Madavai & LC/LA \\
\hline Order 6: Pleuronectiformes & Family 29: Cynoglossidae & 75 & Cynoglossus macrostomus, Norman, 1928 & Malabar tongue sole & Mukuthi athil & NE/LA \\
\hline
\end{tabular}




\begin{tabular}{|c|c|c|c|c|c|c|}
\hline & & 76 & Cynoglossus puncticeps, Richardson, 1846 & Speckled tongue sole & Athil & NE/LA \\
\hline & Family 30: Soleidae & 77 & Euryglossa orientalis, Bloch \& Schneider, 1801 & Oriental tongue sole & Naaku meen & NE/LA \\
\hline \multirow{4}{*}{ Order 7: Siluriformes } & Family 31: Ariidae & 78 & Arius caelatus, Valenciennes, 1840 & Engraved catfish & Venkeluthi & NT/LA \\
\hline & & 79 & Arius tenuispinis, Day, 1877 & Thin spine sea catfish & Kummakeluthi & NT/LA \\
\hline & & 80 & Arius thalassinus, Rüppell, 1837 & Giant catfish & Kattakeluthi & NT/LA \\
\hline & & 81 & Osteogeneiosus militaris, Linnaeus, 1758 & Soldier catfish & Porikeluthi & NE/LA \\
\hline \multirow[t]{3}{*}{ Order 8: Aulopiformes } & Family 32: Synodontidae & 82 & Saurida tumbil, Bloch, 1795 & Greater lizard fish & Thumbili & LC/LA \\
\hline & & 83 & Saurida undosquamis, Richardson, 1848 & Brush tooth lizardfish & Vettanthumbili & LC/LA \\
\hline & & 84 & Harpadon nehereus, Hamillton, 1822 & Bombay duck & Vangaravaasi & NE/VR \\
\hline Order 9: Anguilliformes & Family 33: Muraenesocidae & 85 & Congresox talabonoides, Bleeker, 1853 & Indian pike-conger & Kuliri & NE/VR \\
\hline Order10: Beloniformes & Family 34: Exocoetidae & & & & & \\
\hline \multirow[t]{3}{*}{ Suborder: Exocoetoidei } & Subfamily: Exocoetinae & 86 & Cheilopogon nigricans, Bennett, 1840 & African flying fish & Paravaikola & LC/LA \\
\hline & Subfamily: Hemiramphinae & 86 & Cheilopogon nigricans, Bennett, 1840 & African flying fish & Paravaikola & LC/LA \\
\hline & Family 35: Hemiramphidae & 87 & Hemiramphus far, Forsskål 1775 & Niparus half beak & Oosikola & $\mathrm{LC} / \mathrm{R}$ \\
\hline Order 11: Gadiformes & Family 36: Bregmacerotidae & 88 & Bregmaceros bathymaster, Jordan \& Bollman, 1890 & Codlet & & LC/VR \\
\hline
\end{tabular}

LC- least concern, NT- Near Threatened, VU- Vulnerable, DD- Data Deficient, NE- Not Evaluated, AV-Available, VR- Very Rare, R- Rare, LA- Less Available 


\section{Conclusion}

Biodiversity, the variation of life on Earth, is a major factor in its resilience. It is regularly to be overlooked species that are the most important to healthy ecosystems. There are promising fisheries technologies which have been developed and are being practised for improving fish biodiversity and nutrition. Loss of habitat threatens habitat specialists with extinction risk. The stress on major ecosystems has resulted in erosion of biodiversity due to various anthropogenic activities. This study attempted to record the diversity of commercially important fishes and fishery status of Puducherry coastal waters and also to open up arguments and considerations on the importance of conservation management, and further points to new directions on this frontier

\section{Acknowledgement}

We extend our sincere gratitude to Department of Fisheries \& Fishermen Welfare, Puducherry and NMFDC of CMFRI, Kochi, for providing the information about fishery status. This research did not receive any specific grant from funding agencies in the public, commercial, or not-for-profit sectors.

\section{References}

1. Costello MJ, Bouchet P, Boxshall G, Fauchald K, Gordon DP, Hoeksema BW. Global coordination and standardisation in marine biodiversity through the World Register of Marine Species (WoRMS) and related databases; PLoS one 2013;8:e51629.

2. Mustafa S. Introduction. In: Mustafa S, editor. Genetics in Sustainable Fisheries Management. London: Blackwell Science Publishing 1999, 3-23.

3. Annon. Report of Task Force on Conservation and Sustainable Use of Medicinal Plants. Planning Commission, Government of India 2000.

4. Kestemont P, Didier J, Micah JC, Depiereux E. Selecting Ichthyological metrics to assess river basin ecological quality. Arch. Hydrobiol. Suppl 2000;121:321-348.

5. Schmutz S, Cowx IG, Haidvogal G, Pont D. Fish based methods for assessing European running waters. A synthesis. Fish Mang Ecol 2007;14:369-380.

6. Abell R, Michele L, Thieme ML, Revenga C, Breyer M, Kottelat $\mathrm{M}$ et al. Freshwater ecoregion of the world: a new map of biogeographic units for freshwater biodiversity conservation. Bioscience 2008;58(5):403414.

7. Moyle PB, and Randall PJ. Evaluating the biotic integrity of watersheds in the Sierra Nevada, California. Conservation Biology 1998;12:1318-1326.

8. Jowet IG. In stream low methods: A comparison of approaches. Regul. Rivers: Reg. Manag 1997;13:115127.

9. Moyle PB, Leidy RA. Loss of biodiversity in aquatic ecosystems: Evidence from fish faunas. In: Friedler PL, Jain SK, editors. Conservation Biology. New York: Chapman and Hall 1992, 128-169.

10. Butchart SHM, Walpole M, Collen B, Van Strien A, Scharlemann, JPW, Almon REE. Global biodiversity: Indicators of recent declines. Science 2010;328:11641168.

11. Fischer W, Bianchi G. FAO Species Identification sheets for fishery purposes: Western Indian Ocean (Fishing Area 51) 1984.

12. Grinson George K, Ramani D, Pugazhendi Sindhu K,
Augustine, Sathianandan TV. Marine fish landings in Tamil Nadu and Puducherry during 2016 - An overviewICAR-Central Marine Fisheries Research Institute, Kochi -Madras Research Centre of ICAR-Central Marine Fisheries Research Institute, Chennai 2016.

13. FRAD, CMFRI. Marine Fish Landings in India 2016, Technical Report, CMFRI, Kochi 2017.

14. Banse K. On upwelling and bottom trawling off the south-west coast of India. J Mar. Biol. Assoc. India 1959;1:33-49.

15. Longhurst AR, Wooster WS. Abundance of oil sardine (Sardinella longiceps) and upwelling on the south-west coast of India. Can. J Fish Aquat. Sci 1990;47:2407-19.

16. Madhupratap M, Shetye SR, Nair KNV, Sreekumaran Nair SR. Oil sardine and Indian mackerel, their fishery, problem and coastal oceanography. Curr. Sci 1994;66(5):340-48.

17. Yohannan TM, Abdulrahiman UC. Environmental influence of the behaviour of Indian mackerel and their availability to fishing gear along Malabar Coast. Indian J Fish 1998;45:239-247.

18. Jayaprakash AA. Long term trends in rainfall, sea level and solar periodicity. A case study for forecast of Malabar sole and oil sardine fishery. J. Mar. Biol. Assoc. India 2002;44:163-75.

19. Xu C, Boyce MS. Oil sardine (Sardinella longiceps) off the Malabar Coast: density dependence and environmental effects. Fish. Oceanogr 2009;18(5):359370.

20. Leveque C, Paugy D, Teugels GG. Annotated checklist of the freshwater fishes of the Nilo-Sudan river basin in Africa. Rev. Hydrobiol. Trop 1991;24:131-154.

21. Bassoucalingam Kumaran, Sowjanya Naikar Kambala, Jayaraman Nadarajan. Assessment of Ichthyo-faunal Diversity in Giriyampeta Estuary, Yanam (U.T.of Puducherry). Bull. Environ. Pharmacol. Life Sci 2012;1(9):17-25.

22. Kuppan A, Martin P, Kalaichelvi N, Srinivaasu. S, Sivamani S. Diversity of edible and non-edible marine fishes in East Coastal Region villages at Chennai, Tamil Nadu, India. Int. J Adv. Res. Biol. Sci 2016;3(12):147151.

23. Department of Fisheries \& Fishermen Welfare, Puducherry. Puducherry Fisheries Statistics at a Glance 2010.

24. Konan Grevias N Zi. Stanislas Silvain Yao, Gouli Goore $\mathrm{Bi}$ and valentin Ndouba. Update of ichthyofauna diversity and ecological status of a coastal river NeroWest Africa. Saudi. J Biol 2014;22(3):265-273.

25. Department of Fisheries \& Fishermen Welfare, Puducherry. Puducherry Fisheries Statistics at a Glance 2017.

26. CMFRI. Annual Report 2006-07. Central Marine Fisheries Research Institute, Cochin 2007, 126.

27. Srivastava UK, Dholakia BH, Vathsala S, Chidambaram K. "India Fishery Sector Study", Review and Analysis, IIM-Ahmedabad. Study sponsored by the World Bank through the Min of Food Processing Industries', GoI 1991, 7-12 Annexures

28. Mohamad Kasim H, Vivekanandan V. Marine Fish Production in Tamil Nadu \& Puducherry. A Report based on a detailed analysis of Central Marine Fisheries Research Institute Data 2011.

29. Imtiyaz BB, Sweta PD, Prakash KK. Threats to Marine 
Biodiversity. Marine Biodiversity: Present Status and Prospects 2011, 21-26.

30. Dulvy NK, Fowler S, Musick SJ, Cavanagh RD. Kyne PM, Harrison LR et.al. Extinction risk and conservation of the world's shark and rays. eLIFE 2014;3:e00590. https://sites.google.com/site/fimsul/

31. IUCN Red List of Threatened Species, Version 2016. http://www.iucnredlist. Org/search, IUCN.

32. Ramachandran S. Coastal Environment and Management. Institute of Ocean Management, Anna University, Chennai 2001, 347. 